\title{
Dispneia Como Manifestação Inicial de Doença Rara
}

\section{Dyspnea as Manifestation of a Rare Disease}

Margarida Gaudêncio ${ }^{1 *}$, Joana Antunes², Ivo Barreiro² ${ }^{2}$ Maria Inês Bertão², Isabel Bessa ${ }^{3}$, Amélia Pereira ${ }^{4}$

\section{RESUMO}

A doença de Madelung é uma condição rara, caracterizada pelo depósito maciço de gordura. Esta doença é conhecida como lipomatose simétrica múltipla ou benigna. Encontram-se descritos dois tipos de doença. No tipo 1, a gordura acumula-se no pescoço, ombros, membros superiores e tronco superior. No tipo 2, os lipomas distribuem-se por todo o corpo, incluindo nos membros inferiores. Os doentes geralmente referem algum desconforto, no entanto, em casos avançados, os lipomas podem causar sintomas como dispneia ou disfagia.

Os autores apresentam o caso clínico de um doente do género masculino de 70 anos, referenciado para o Serviço de Urgência por dispneia com alguns meses de evolução, com agravamento nos últimos 6 dias, associada a tosse. Ao exame objetivo, destacava-se a presença de lipomas a nível da região cervical e torácica, com distribuição simétrica e circulação venosa colateral. Em angio-tomografia computorizada (TC) cervical e torácica, evidenciou-se a presença de proliferação da gordura cervical simétrica sugestiva de doença de Madelung, com compressão da veia jugular e braquiocefálica direita, assim como, formação de veias colaterais e consolidação peribrônquica dos lobos inferiores e médio com broncograma aéreo. Admitido no Serviço de Medicina para antibioterapia e oxigénio suplementar. Apresentou uma evolução clínica e analítica favoráveis, tendo alta ao quinto dia de hospitalização, orientado para consulta de Cirurgia.

O caso apresentado corresponde a uma doença de Madelung tipo 1, em que o envolvimento mais profundo levou ao desenvolvimento de dispneia, agravado por intercorrência infeciosa.

1. Interno de Medicina Interna, Serviço de Medicina Interna do Hospital Distrital da Figueira da Foz, Figueira da Foz, Portugal. 2. Interno de Formação Específica de Medicina Interna, Serviço de Medicina Interna do Hospital Distrital da Figueira da Foz, Figueira da Foz, Portugal. 3. Assistente Graduado de Medicina Interna, Serviço de Medicina Interna do Hospital Distrital da Figueira da Foz, Figueira da Foz, Portugal. 4. Assistente Graduado Sénior de Medicina Interna, Serviço de Medicina Interna do Hospital Distrital da Figueira da Foz, Figueira da Foz, Portugal. 
O diagnóstico desta patologia é baseado sobretudo na história clínica, exame objetivo (com a presença de múltiplos lipomas simétricos) e estudo imagiológico. A etiologia desta patologia permanece desconhecida. Atualmente, a cirurgia é o único tratamento disponível, apesar da elevada taxa de recorrência.

PALAVRAS-CHAVE: Dispneia; Lipomatose Simétrica Múltipla

\section{ABSTRACT}

Madelung's disease is a rare condition characterized by a symmetrical and painless pattern of massive fatty deposits. The condition is also known as benign or multiple symmetric lipomatosis. Two types of this disease have been described. In type 1, fat accumulates around the neck, shoulders, upper arms and upper back. In type 2, lipomas are distributed over much of the body, including the hips and thighs. The patients complain of their appearance although, in advanced cases, the increase in lipomas could cause symptoms as dyspnea or dysphagia.

The authors propose to present the clinical case of a 70-year-old male patient referred to the emergency department for dyspnea with a few months of evolution, with worsening in the last 6 days, associated with cough. At physical examination, had no changes, except the presence of lipomas at the cervical and upper thoracic and back levels, with symmetrical distribution and collateral venous circulation. Computed tomography (CT) angiography of neck and thorax have shown proliferation of cervical fat suggestive of Madelung's disease, with compression of the jugular veins and right brachiocephalic vein, as well as formation of collateral veins and a peribronchial consolidation in the lower and middle lobes with air bronchogram. He was hospitalized for oxygen and empirically antibiotic therapy. He had a good clinical and analytical evolution and was discharged after 5 days of hospitalization, oriented to Surgery's consultation.

The case presented corresponds to a Madelung's disease type 1, in which deep involvement conditioned the onset of dyspnea exacerbated by infectious intercurrence.

The diagnosis of Madelung's disease is primarily based on clinical history, physical examination and imaging studies. The clinical diagnosis is based on the recognition of the unique appearance of the patient. The etiology of this disease remains unknown. Currently, surgery is the only treatment available, despite the high recurrence rate.

KEYWORDS: Dyspnea; Lipomatosis, Multiple Symmetrical

\section{INTRODUÇÃO}

A lipomatose simétrica múltipla ou doença de Madelung é uma doença benigna e rara da diferenciação adipocitária, caracterizada por lipomatose difusa sobretudo na região cefálica, cervical e torácica superior. ${ }^{1}$ Os doentes apresentam-se desta forma, com múltiplos lipomas, indolores e de consistência mole à pal pação, sendo que, ao contrário da maioria dos lipomas subcutâneos isolados, o tecido lipomatoso nesta entidade não é encapsulado, tendo por isso, a capacidade de infiltrar espaços entre estruturas subcutâneas e musculares adjacentes. ${ }^{2}$

Em termos epidemiológicos, é desconhecida a verdadeira incidência da lipomatose simétrica múltipla, no entanto, a maioria das séries relatadas constatou que esta entidade ocorre mais frequentemente na bacia do Mediterrâneo, especialmente em Itália, onde se estima uma incidência de cerca de 1:25 000. ${ }^{3}$ Nos últimos anos, têm sido descritos igualmente alguns casos, em menor número no Norte da Europa, Europa de Leste e em paí- ses como Israel, China, Estados Unidos da América, Brasil, Argentina e México. ${ }^{4}$

A doença de Madelung parece ser uma entidade primária e específica do tecido adiposo, resultando de um distúrbio metabólico dos lípidos, contudo, ainda permanecem desconhecidos os mecanismos fisiopatológicos subjacentes. $^{5}$

De modo geral, estes doentes recorrem ao médico mais frequentemente por uma questão estética. ${ }^{6}$ No entanto, podem apresentar outros sintomas, nomeadamente relacionados com a presença de neuropatia periférica em cerca de $85 \%$ dos doentes. ${ }^{7}$ Mais raramente, em virtude das massas lipomatosas serem não encapsuladas, pode ocorrer infiltração de estruturas da região cervical e originar, desta forma, compressão das vias aéreas, sistema digestivo e provocar sintomas como dispneia e/ ou disfagia, assim como, condicionar síndrome de apneia obstrutiva do sono. ${ }^{2}$ 
O diagnóstico desta patologia é clínico e realizado com base na história clínica e exame físico.2,6

Os autores propõem-se apresentar um caso de doença de Madelung, no qual, o principal sintoma do doente era dispneia.

\section{CASO CLÍNICO}

Doente de 70 anos do género masculino, caucasiano, recorreu ao Serviço de Urgência por dispneia com alguns meses de evolução, no entanto, com agravamento 6 dias antes, associada a tosse seca.

Os antecedentes pessoais eram irrelevantes exceto a presença de hábitos etílicos moderados. Sem história de

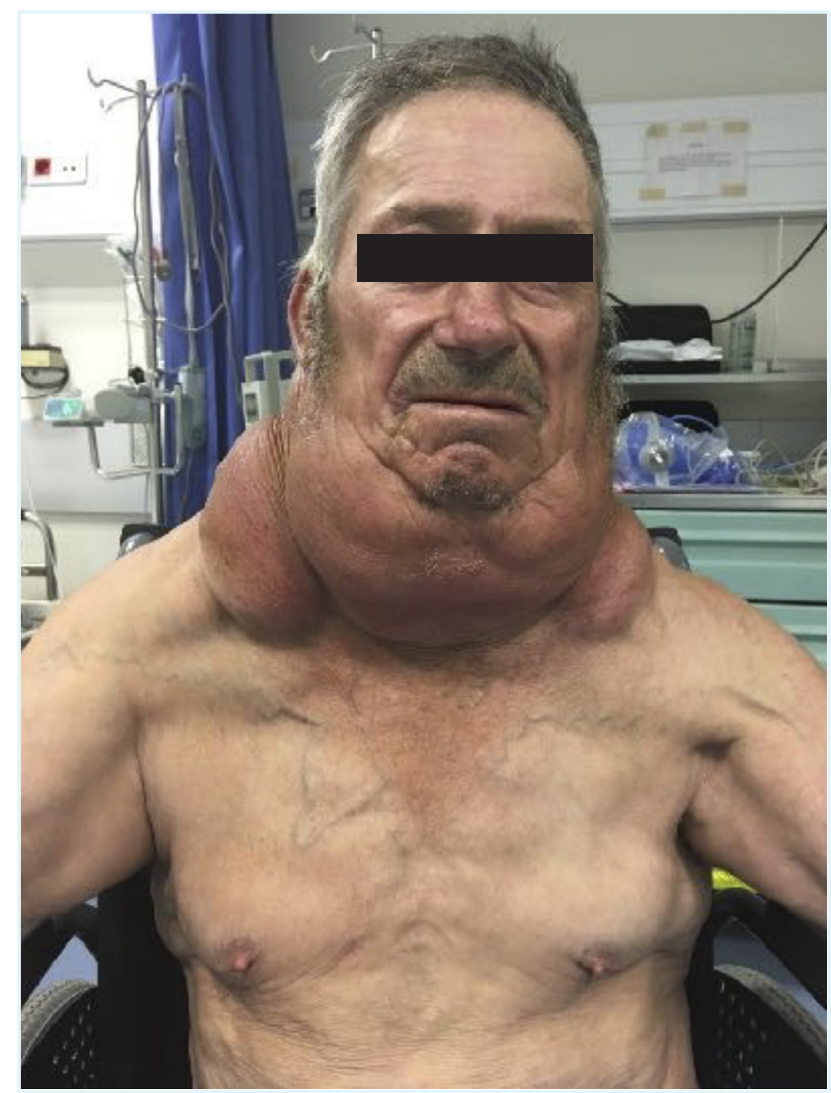

FIGURA 1. Lipomatose múltipla simétrica, sobretudo na região cervical e torácica superior e anterior.

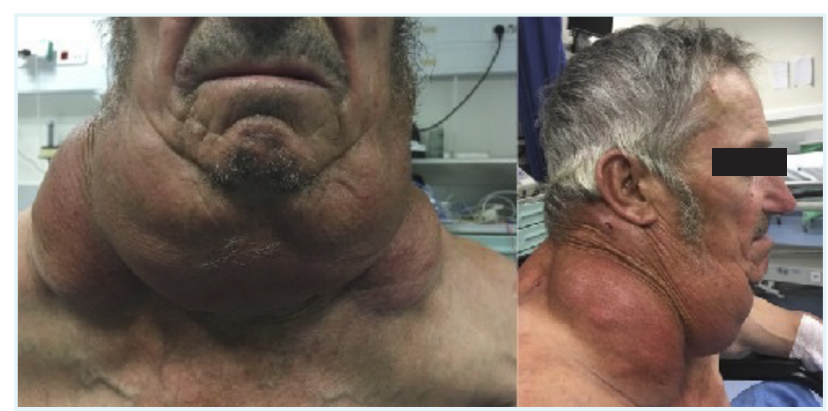

FIGURA 2. Lipomatose múltipla simétrica com distribuição na região cervical. alergias conhecidas. Segundo informação obtida, teria realizado excisão de lipomas há vários anos, no entanto, não teria qualquer seguimento nem diagnóstico estabelecido. Sem hábitos medicamentosos.

Nos antecedentes familiares, desconheciam-se histórias de patologias de relevo, nomeadamente hereditárias.

No exame objetivo, à admissão hospitalar, o doente encontrava-se consciente, orientado e colaborante, hemodinamicamente estável, eupneico em ar ambiente, com saturação periférica de $\mathrm{O}_{2}$ de 93\%. À auscultação cardíaca e pulmonar, não apresentava alterações. Do restante exame, destacava-se a presença de lipomatose cervical e torácica superior, bilateralmente, simétrica (Fig.s 1 a 3) e com circulação venosa colateral (Fig. 4).

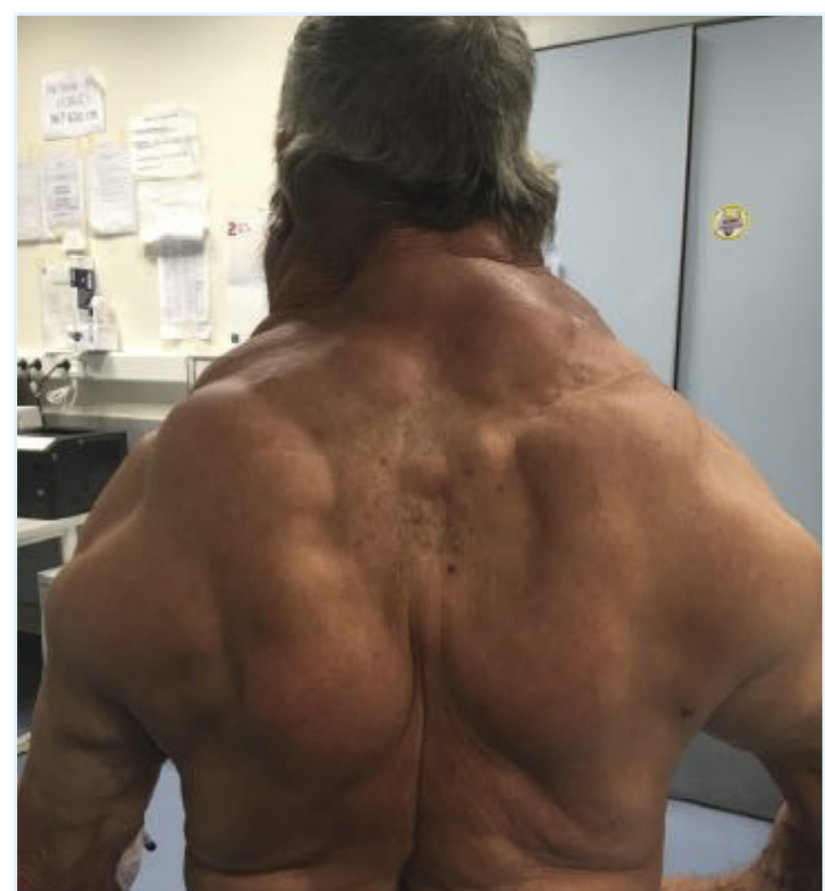

FIGURA 3. Múltiplos lipomas dispersos simetricamente na região torácica superior e posterior.

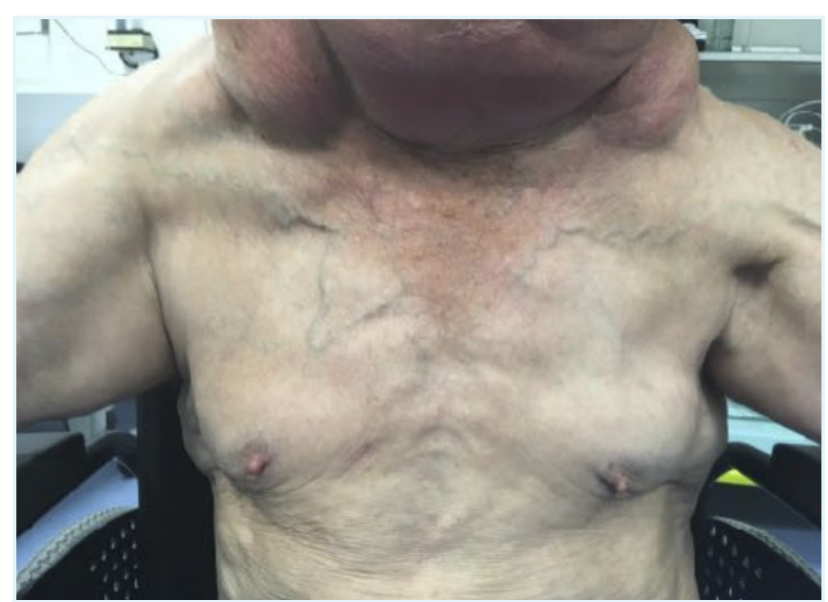

FIGURA 4. Circulação venosa colateral na região torácica ântero-superior, bilateralmente. 
Analiticamente, objetivou-se anemia macrocítica com hemoglobina de 13,3 g/dL e volume globular médio de 103 fL ( N 78-100 fL), D-dímeros 614 ng/mL ( $<243$ $\mathrm{ng} / \mathrm{mL}$ ) e elevação da proteína C reativa de 79,41 mg/L $(\mathrm{N}<5 \mathrm{mg} / \mathrm{L})$. Em termos gasimétricos, apresentava $\mathrm{pH}$ de 7,467, $\mathrm{pO}_{2}$ de 64,2 $\mathrm{mmHg}, \mathrm{pCO}_{2}$ de 28,7 $\mathrm{mmHg}$, $\mathrm{HCO}_{3} 20,5$, lactatos de 2,4 mmol/L.

Realizou angio-tomografia computorizada (TC) cervical e torácica, verificando-se proliferação marcada da gordura cervical, permeando os músculos cervicais posteriores e esternocleidomastoideus, bilateralmente, bem como, compressão das veias jugulares pelas massas adiposas (Fig. 5) e ausência de massas cervicais de caráter expansivo. Nas secções torácicas, ausência de sinais sugestivos de tromboembolismo pulmonar, no entanto, destacava-se a presença de focos de consolidação peribrônquica nos lobos inferiores e lobo médio direito, com broncograma aéreo, sugerindo patologia infeciosa, assim como compressão ao nível da veia braquiocefálica direita também por massas adiposas (Fig. 6) e ausência de adenopatias ou outras massas expansivas torácicas.

Foi internado no Serviço de Medicina por pneumonia lobar e insuficiência respiratória tipo 1 com necessidade de oxigenoterapia suplementar.

Durante o internamento, verificou-se uma melhoria gradual da sintomatologia, bem como, apirexia sustentada e estabilidade hemodinâmica, com diminuição progressiva das necessidades de oxigenoterapia. $\bigcirc$ doente cumpriu antibioterapia empírica com amoxicilina/ácido clavulânico e claritromicina, completando um esquema de
7 dias de terapêutica. Analiticamente, o doente apresentou uma redução sustentada dos parâmetros infeciosos.

Dada a melhoria clínica e analítica, o doente teve alta ao $5^{\circ}$ dia de internamento, tendo sido orientado para consulta de Cirurgia para excisão de lipomas, sobretudo a nível cervical. No período de follow-up, embora o doente tenha recusado tratamento cirúrgico, com a cessação dos consumos etílicos, não se objetivou um aumento dimensional dos lipomas nem surgiram novas lesões, sobretudo na região cervical.

\section{DISCUSSÃO E CONCLUSÃO}

A lipomatose simétrica benigna foi descrita inicialmente por Sir Benjamin Brodie em 1846, em Londres, no St. George Hospital. ${ }^{4}$ No entanto, a aparência física de uma estátua de um guerreiro Capestrano encontrada em Itália, na região de Abruzzi, e que remonta ao século VI, parece sugerir a existência desta doença em tempos remotos. $^{4}$

Esta patologia é considerada uma entidade rara, que se caracteriza pela acumulação de múltiplos depósitos de tecido adiposo, não encapsulado, distribuídos de forma simétrica em várias localizações anatómicas, com um forte predomínio da região cefálica, cervical, supra-escapular, supra-clavicular, parede torácica superior, região abdominal e inguinal, assim como, extremidade proximal dos membros superiores e inferiores. ${ }^{2}$

Em termos de classificação, a lipomatose simétrica múltipla ou benigna pode dividir-se em tipo 1 (lipomatose difusa do pescoço, região cervical e torácica superior)

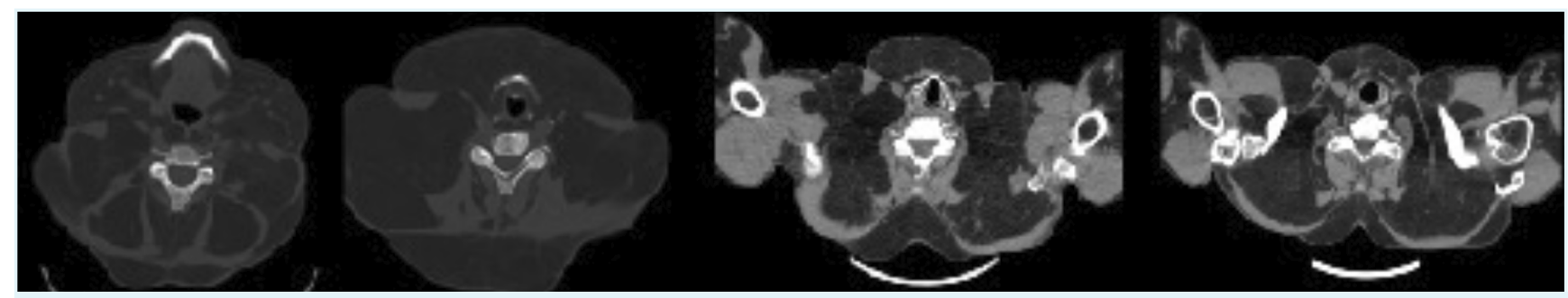

FIGURA 5. Cortes transversais de angio-TC cervical demonstrando proliferação marcada da gordura cervical, permeando os músculos cervicais posteriores e esternocleidomastoideus e compressão das veias jugulares por massas adiposas.

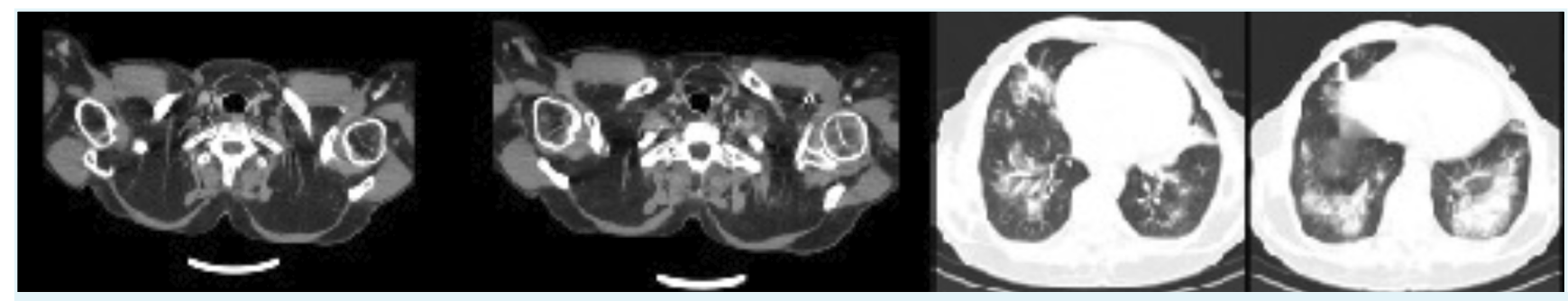

FIGURA 6. As duas primeiras imagens correspondem a cortes transversais de angio-TC torácica, demonstrando compressão a nível da veia braquicefálica direita por massas adiposas. As duas últimas imagens correspondem a cortes transversais de TC torácica em janela de pulmão, demonstrando focos de consolidação peribrônquica nos lobos inferiores e lobo médio direito com broncograma aéreo, sugerindo patologia infeciosa. 
e tipo 2 (lipomatose difusa, simétrica com distribuição por todo o corpo, incluindo nos membros inferiores). ${ }^{8}$ Donhauser et al reconhecem um terceiro fenótipo desta patologia que se caracteriza por lipomatose difusa sobretudo na região das ancas e região superior das coxas. ${ }^{9}$

A doença de Madelung parece ter uma maior incidência em indivíduos de raça caucasiana, embora existam relatos em doentes de outras raças. ${ }^{8}$ Em termos de género, parece ser mais prevalente no sexo masculino, tendo-se verificado no género feminino numa proporção que varia entre 1:15 e 1:30.7 A variante mais comum é o tipo 1, com maior incidência no género masculino, no entanto, no sexo feminino é mais frequente o tipo 2, uma vez que, apresenta um padrão de distribuição mais difuso e acompanha-se frequentemente de um índice de massa corporal elevado.? Esta entidade tipicamente ocorre entre a $3^{\text {a }}$ e $6^{\text {a }}$ década de vida. ${ }^{1}$

Apesar da etiologia e patogenia da lipomatose múltipla serem desconhecidas, a maioria dos estudos aponta uma associação quase absoluta com o alcoolismo crónico, sendo que, mais de 90\% dos doentes apresenta história de ingestão crónica moderada a elevada de álcool. ${ }^{2}$ Embora se encontre descrito uma ligeira regressão dos lipomas com a abstinência etílica e um crescimento acelerado dos mesmos com o aumento da ingestão, a doença não entra em remissão com a cessação total do consumo de bebidas alcoólicas. ${ }^{2}$ Numa minoria dos doentes estão descritas mutações no DNA mitocondrial, sendo que, Perera et al descrevem uma série de casos de doença de Madelung numa família associados a mutação no gene mitocondrial MTTK variante c.8344A $>6 .{ }^{1}$

A maioria dos casos de doença de Madelung são esporádicos, contudo, embora não seja uma doença caracteristicamente hereditária, já foram observados alguns casos familiares, a maioria dos quais com transmissão autossómica dominante. ${ }^{2}$ No entanto, a base genética da hereditariedade nesta doença permanece ainda por elucidar; como tal, os casos hereditários são geralmente designados de lipomatose múltipla familiar. ${ }^{2}$

A lipomatose simétrica múltipla apresenta um quadro clínico muito característico, sobretudo devido à deformação estética que provoca nos doentes. ${ }^{8}$ Como a maioria dos indivíduos são assintomáticos, para além da aparência física característica, não é obrigatória a presença de outros sinais ou sintomas para o diagnóstico desta doença. ${ }^{8}$

Na maioria dos casos, o uso de exames complementares para fins diagnósticos pode ser dispensável, uma vez que, em 85\% dos doentes, o exame físico e história clíni- ca são suficientes para o diagnóstico clínico de lipomatose simétrica múltipla. ${ }^{8}$

Quando sintomas como a dispneia e/ou disfagia surgem e são o motivo pelo qual os doentes recorrem ao médico, é necessário excluir sempre a hipótese de etiologia maligna subjacente, sendo necessário recorrer a exames complementares como a tomografia computorizada, ressonância magnética e exames endoscópicos. ${ }^{3}$

Em termos de avaliação laboratorial, não existem alterações específicas para esta doença, contudo, tem sido associada à existência de um padrão metabólico característico, ou seja, com elevação dos níveis de colesterol HDL (por aumento da produção) e concentrações mais baixas de colesterol LDL. ${ }^{6}$

A utilização de exames de imagem como a ecografia, tomografia computorizada e ressonância magnética pode ser considerada para a confirmação da presença de massas com vista ao diagnóstico de lipoma e para exclusão de malignidade. ${ }^{8}$ No entanto, a investigação adicional está indicada nos casos de suspeita de malignidade ou na presença de lipomas de grandes dimensões que condicionam efeito de massa e compressão de estruturas do pescoço, procurando desta forma, avaliar a extensão da infiltração da massa lipomatosa e determinar qual a melhor opção terapêutica (avaliação pré-operatória). ${ }^{8}$

O diagnóstico definitivo é determinado histologicamente pela visualização do conteúdo das massas lipomatosas. ${ }^{10}$ Para além de definir os subtipos histológicos dos lipomas, a biópsia é o único método complementar de diagnóstico que permite excluir ou diagnosticar definitivamente a malignidade. ${ }^{10}$ Em suma, devem ser submetidos a biópsia todos os casos de doença de Madelung que forem sujeitos a excisão de lipomas ou nos casos de suspeita de massa não lipomatosa. ${ }^{10}$

A principal complicação da lipomatose simétrica múltipla é a deformação estética e funcional que as massas lipomatosas condicionam. ${ }^{11}$ No entanto, as complicações mais graves derivam da compressão das vias aéreas e digestiva, compressão do nervo laríngeo recorrente e das estruturas mediastínicas, reproduzindo uma síndrome da veia cava superior. ${ }^{11}$ É igualmente de salientar, que estão descritas alterações do sistema nervoso em mais de 50\% dos casos, sendo que, a manifestação neurológica mais estudada é a neuropatia periférica. ${ }^{11}$ Outra das complicações descrita na literatura, apesar de ser rara, é a degeneração maligna das massas lipomatosas. ${ }^{3}$

Em termos de terapêutica, tem sido proposto um número considerável de medidas conservadoras e de tratamento médico para esta doença, mas nenhuma delas mostrou ter um efeito terapêutico definitivo. ${ }^{8}$ Uma des- 
tas medidas é a abstinência etílica, uma vez que, apesar de não induzir a regressão dos lipomas, parece prevenir a progressão e crescimento adicional dos mesmos. ${ }^{8}$ Outra das medidas recomendadas passa pela perda ponderal, apesar de não ter mostrado efeito nas massas lipomatosas, permite diminuir o risco metabólico global destes doentes, que constitui um dos principais fatores de mau prognóstico. ${ }^{8}$ Em suma, os autores consideram que novas opções terapêuticas serão desenvolvidas quando a etiopatogenia desta doença for completamente esclarecida.

Na atualidade, o tratamento de eleição é a cirurgia, mas que está reservada apenas para doentes com deformações estéticas, envolvimento funcional ou compromisso de estruturas vitais, uma vez que, a recorrência é muito comum e está na base da falta de encapsulamento das massas, elevada vascularização das mesmas e presença de estroma fibroso na sua constituição, o que torna a excisão completa dos lipomas extremamente difícil. ${ }^{11}$ A lipectomia e a lipoaspiração são as modalidades cirúrgicas que têm sido propostas para a excisão dos lipomas. ${ }^{5}$ A lipoaspiração tem apresentado algumas vantagens quando comparada com a técnica convencional, nomeadamente menor risco geral, redução do tempo operatório, menor número de efeitos adversos, melhores resultados estéticos e ausência de limitação no que diz respeito ao tamanho dos lipomas. ${ }^{5}$

Por fim, em termos de prognóstico, a doença de Madelung é uma entidade lentamente progressiva com um curso e evolução relativamente benignos. ${ }^{6}$ A história natural desta patologia é caracterizada por uma substancial morbilidade, uma vez que, a compressão, desvio e infiltração de estruturas como as vias aéreas, esófago e mediastino são responsáveis por dispneia, disfagia e síndrome de apneia obstrutiva do sono, que ocorrem em cerca de 40\% dos doentes, acarretando pior prognóstico e morbilidade acrescida, pelo que, nestes casos, a remoção cirúrgica dos lipomas é quase mandatória. ${ }^{6}$

Neste caso, o doente apresentava múltiplos lipomas dispersos sobretudo na região cervical e torácica superior com alguns anos de evolução, no entanto, sem limitação funcional, pelo que, o doente não tinha seguimento prévio, tendo feito excisão de alguns lipomas, mas com recorrência. Contrariamente à maioria dos doentes que recorre ao médico por uma questão estética, este doente recorreu ao Serviço de Urgência por dispneia com algum tempo de evolução, no entanto, com um agravamento recente.

Mesmo com uma história clínica (incluindo consumos etílicos) e exame objetivo altamente sugestivos de doen- ça de Madelung, a equipa médica optou por realizar estudo complementar com tomografia computorizada, uma vez que, uma das hipóteses diagnósticas colocadas foi a de síndrome da veia cava superior secundária à compressão externa por massas adiposas pela presença concomitante de circulação venosa colateral. No entanto, o que se verificou foi uma compressão das veias jugulares e braquiocefálica direita que justificam a rede venosa colateral objetivada e, por outro lado, a realização deste exame complementar permitiu igualmente o diagnóstico de uma pneumonia lobar, que neste caso, veio agravar a sintomatologia já existente.

À data de alta, o doente foi encaminhado para consulta de Cirurgia com vista a avaliação e ponderação de eventual excisão de lipomas, dada a compressão externa venosa diagnosticada. No entanto, recusou intervenção cirúrgica, optando por cessar consumos etílicos. Com a abstinência etílica, verificou-se um atraso na progressão, ou seja, não se verificou o aparecimento de novas lesões, sobretudo a nível cervical, com melhoria dos sintomas compressivos, nomeadamente dispneia. Contudo, uma vez que, não foi submetido a intervenção cirúrgica, não se observou uma regressão total dos sinais de compressão venosa, mantendo vigilância em consulta.

\section{RESPONSABILIDADES ÉTICAS}

CONFLITOS DE INTERESSE: Os autores declaram a inexistência de conflitos de interesse na realização do presente trabalho.

FONTES DE FINANCIAMENTO: Não existiram fontes externas de financiamento para a realização deste artigo.

CONFIDENCIALIDADE DOS DADOS: Os autores declaram ter seguido os protocolos da sua instituição acerca da publicação dos dados de doentes.

CONSENTIMENTO: Consentimento do doente para publicação obtido.

PROVENIÊNCIA E REVISÃO POR PARES: Não comissionado; revisão externa por pares.

\section{ETHICAL DISCLOSURES}

CONFLICTS OF INTEREST: The authors have no conflicts of interest to declare.

FINANCING SUPPORT: This work has not received any contribution, grant or scholarship.

CONFIDENTIALITY OF DATA: The authors declare that they have followed the protocols of their work center on the publication of data from patients. 
PATIENT CONSENT: Consent for publication was obtai-

ned.

PROVENANCE AND PEER REVIEW: Not commissioned; externally peer reviewed.

\section{REFERÊNCIAS}

1. Perera U, Kennedy BA, Hegele RA. Multiple Symmetric Lipomatosis (Madelung Disease) in a Large Canadian Family with the Mitochondrial MTTK c.8344A>G Variant. J Investig Med High Impact Case Rep. 2018;6:2324709618802867. doi: 10.1177/2324709618802867.

2. Enzi G, Busetto L, Ceschin E, Coin A, Digito M, Pigozzo S. Multiple symmetric lipomatosis: clinical aspects and outcome in a long-term longitudinal study. Int J Obes Relat Metab Disord. 2002;26:253-61. doi: 10.1038/sj.ijo.0801867.

3. Nemi S, Maxit M. Lipomatosis Simétrica Múltiple (Enfermedad de Madelung). Rev Hosp Privado Comunidad.2003;6:58.

4. Mayor M, Arillo A, Tiberio G.2006, 'Lipomatosis simétrica multiple: a propósito de un caso. Sist Sanit Navar. 2006;29:433-7.

5. Chen CY, Fang QQ, Wang XF, Zhang MX, Zhao WY, Shi BH, et al. Madelung's Disease: Lipectomy or Liposuction? Biomed Res Int. 2018;2018:3975974. doi: 10.1155/2018/3975974.

6. Dökmetas HS, Korkmaz S, Ozcelik S, Koyuncu A. Madelung's disease in a patient with diabetes mellitus. Skinmed. 2007;6:247-9. doi: 10.1111/j.1540-9740.2007.05936.x.

7. Busetto L, Sträter D, Enzi G, Coin A, Sergi G, Inelmen EM, et al. Differential clinical expression of multiple symmetric lipomatosis in men and women. Int J Obes Relat Metab Disord. 2003;27:1419-22. doi: 10.1038/sj.ijo.0802427.

8. Rodrigues L. Madelung's disease: a case report and literature review. Radiol Brasil. 2012;45:129-131.

9. Harsch IA, Schahin SP, Fuchs FS, Hahn EG, Lohmann T, Konturek $\mathrm{PC}$, et al. Insulin resistance, hyperleptinemia, and obstructive sleep apnea in Launois-Bensaude syndrome. Obes Res. 2002;10:625-32. doi: 10.1038/oby.2002.85.

10. Roberts CC, Liu PT, Colby TV. Encapsulated versus nonencapsulated superficial fatty masses: a proposed MR imaging classification. AJR Am J Roentgenol. 2003;180:1419-22. doi: 10.2214/ajr.180.5.1801419.

11. Sánchez MD, Rosendo MV, Alvarez CP, Caaveiro LP. Lipomatosis simétrica multiple: presentatación de dos formas clínicas diferentes y revisión de literatura. Med Intern. 2006;20:2436. 